\title{
Corela
}

Cognition, représentation, langage

HS-8 | 2010

L'interpellation

\section{L'Interpellation : un objet discursif singulier... et pluriel}

Frédéric Torterat et André Thibault

\section{OpenEdition}

\section{Journals}

Édition électronique

URL : http://journals.openedition.org/corela/798

DOI : $10.4000 /$ corela.798

ISSN : $1638-573 \mathrm{X}$

\section{Éditeur}

Cercle linguistique du Centre et de I'Ouest - CerLICO

Référence électronique

Frédéric Torterat et André Thibault, «L'Interpellation : un objet discursif singulier... et pluriel », Corela [En ligne], HS-8 | 2010, mis en ligne le 23 novembre 2010, consulté le 30 avril 2019. URL : http:// journals.openedition.org/corela/798; DOI : 10.4000/corela.798

Ce document a été généré automatiquement le 30 avril 2019

\section{(c) (i) (2)(2)}

Corela - cognition, représentation, langage est mis à disposition selon les termes de la licence Creative Commons Attribution - Pas d'Utilisation Commerciale - Partage dans les Mêmes Conditions 4.0 International. 


\title{
L'Interpellation : un objet discursif singulier... et pluriel
}

\author{
Frédéric Torterat et André Thibault
}

\section{Un cadre général}

1 Les contributions regroupées dans le présent volume se rassemblent autour d'un même objet, l'interpellation, lequel renvoie à plusieurs types de concrétisations discursives. Ainsi peut-on lui faire correspondre des formules allocutives, des constructions verbales, mais aussi, à certains égards, des registres ou des genres discursifs.

2 Conformément à la principale acception de son correspondant latin, l'interpellation se caractérise surtout par l'action d'interrompre, et par ce qui s'interpose, de manière plus ou moins intempestive, en particulier entre les discours et les personnes. De ce fait, l'interpellation intègre des processus de gradation, d'intensité, autant qu'elle s'inscrit dans un cadre plus ou moins allocutif. Au sens linguistique, cette notion, dont la judiciarisation n'a rien d'un automatisme, renvoie toutefois à un certain nombre d'opérations et de catégories qu'il convient de positionner par rapport aux champs de l'apostrophe et des interjections (ainsi que du vocatif), mais aussi à ceux, plus généraux, de la nomination (Noailly 1995, Détrie 2007), de la désignation/référence (Schegloff, in Stivers \& Enfield 2007) et de la topicalisation (Lambrecht 1998 inter al.). De même, si l'on se place du point de vue de la structure prédicative des énoncés, le positionnement de l'interpellation en termes de prédication complémentaire mérite d'être consulté plus avant, d'autant qu'éventuellement envisagée comme telle dans les relations qu'elle entretient avec les appellatifs (Hammermüller 1997, Pop 2000), les désignations connotées (Lagorgette 2003, Lopez-Muñoz, Marnette et Rosier 2004, O'Kelly 2005) et les constructions appositives (Forsgreen, Jonasson et Kronning 1998, Neveu 2000), celle-ci apparaît comme un objet de recherche à la fois pluriel et pour le moins particulier.

3 Dans une perspective dialogique, l'interpellation implique une manière de rendre le destinataire présent non seulement dans ce qui réfère à lui, mais aussi dans la forme ellemême que prend le discours qui l'interpelle. En effet, les travaux sur l'interaction ont 
contribué, depuis les années 1960, à la prise en compte de la manière dont l'interpellé est désigné (Schegloff 1979), mais aussi à celle du propos qui lui est adressé (recipient designed ). Dans cette vue, il paraît opportun d'envisager les cas où l'interpellation appartient à un certain " cadre figuratif », par le biais notamment de l'intimation, catégorie dans laquelle Benveniste place les ordres, ainsi que les appels «impliquant un rapport vivant et immédiat de l'énonciateur à l'autre" (1977, V), ce qui n'est pas sans rappeler le rapprochement qu'effectue Charaudeau (2002) entre interpellation et identification. D'autre part, il importe sans doute d'apporter quelques éléments de réponse, même de manière intermédiaire, sur la portée illocutoire, et dans tous les cas socio-pragmatique de l'interpellation. Pour autant, ces questionnements en sous-tendent d'autres, notamment à travers ce que soulève l'interpellation d'un point de vue sociolinguistique. Comme le rappelle R. Vion par ailleurs $(1999,98)$, " prévenir une objection, flatter le lecteur, le prendre à témoin, l'interpeller, chercher à le convaincre ou le séduire, raconter ou décrire ne peuvent être que des activités fondamentalement interlocutives. Dans ces conditions, toute forme discursive repose sur la mise en œuvre d'un certain mode d'interlocution et d'un certain type de relation sociale ».

4 En contexte didactique, l'interpellation présente des enjeux tout à fait spécifiques. Action courante de ce que l'on pourrait appeler la "vie de classe", avec l'ensemble des comportements qu'elle implique, l'interpellation pose là aussi la question d'une éventuelle gradation, ne serait-ce que sur le plan de ce qu'on appelle communément, et quelquefois par euphémisme, "l'oral désordonné». Représentée dans sa dimension interlocutive, l'interpellation s'inscrit à ce titre dans un certain schéma participatif (avec ses paramètres de distanciation et de proximité). En plus du fait qu'elle participe d'actions verbales témoignant de certaines phases d'acquisition (Morrison et Ellis 1995, Spieler et Balota 2000), elle suppose aussi une réflexion sur les formats de participation existant dans certaines classes d'accueil. Bien que le colloque auquel renvoient les présentes contributions n'ait pas conduit les intervenants à insister sur ce propos, il est par ailleurs possible de lui reconnaître des contours intonatifs récurrents à l'oral, ce qui semble significatif dans le cas des sommations interpellatives par exemple.

Dans cette vue, le présent volume soumet la question non pas à travers ce qui la résume, mais ce qui la positionne dans les dimensions, multiples, qu'elle occupe. C'est pourquoi nous avons tenté, à travers un titre énumératif et ouvert, de suggérer ce qui permet avant tout d'en envisager la diversité, comme c'est le cas à travers la prédication d'abord, dont il est possible de donner plusieurs versions, mais dont nous retiendrons ici plus particulièrement ce qu'en déduisent F. Rastier et F. Jacques. Rastier (1998) caractérise effectivement la prédication comme "polyadique », et l'envisage dans le cadre d'une anthropologie sémiotique, en lui assignant plusieurs instances qu'elle met en relation. De ce fait et d'après l'auteur, "le contexte et le texte proposent des matérialisations de parcours (et non des instanciations de variables)» (459), ce qui se vérifie vraisemblablement pour l'interpellation et ce qui fait d'elle, à proprement parler, un événement discursif. De son côté, Jacques (2007) confirme que «la prédication ne fonctionne que sous la dépendance d'un certain acte énonciatif. L'énoncé n'est pas seulement par son énonciation un fait, il est également le produit d'un faire. Autrement dit, la dimension événementielle elle-même se spécifie comme acte d'énonciation » (52).

6 Les questions des récurrences et des formes discursives, de leur côté, ont surtout pour objet d'indiquer une tension entre individuation et pluralisation, mais aussi entre singularisation et généralisation. Bien que le terme de formation discursive nous ait paru 
approprié dans un premier temps, en ce qu'il transcende notamment les formes de textualité, mais aussi les domaines ou les (sous-)genres discursifs, il ne résume pas à lui seul ce qu'entendent démontrer la plupart des contributeurs à ce volume. Le terme, attribuable à Foucault et remanié par Haroche, Henry et Pêcheux (1971), lesquels s'en saisissent pour représenter la "place» sociale des énonciateurs à travers leur attachement idéologique, concerne des pratiques discursives qui présentent une certaine régularité, mais qui interviennent, en dernière analyse, dans des cas spécifiques (Courtine 1981, Achard 1995). Les formations discursives impliquent des ressemblances qui témoignent surtout, comme le résume Mayaffre (2004), « du positionnement idéologicosocial de l'instance énonciative ", d'autant qu'elles contribuent à la fois à structurer les cadres sociaux, et les discours eux-mêmes, comme l'a rappelé tout dernièrement Sassier (2008). Nous avons ainsi écarté ce terme pour l'expression, plus généralisable, de récurrences discursives. Celles-ci rejoignent en grande partie ce que C. Détrie, P. Siblot et B. Vérine appellent les "régularités discursives ", à ceci près que les récurrences renvoient plus ouvertement (et plus simplement) à ce qui revient de production discursive en production discursive, quels qu'en soient les contextes. Est récurrent ce qui se répète, ce qui se reproduit, mais aussi ce qui revient en arrière. A ce titre et notamment dans l'histoire des sciences, l'approche des récurrences permet de ne pas réduire l'analyse à un récit d'événements ou à la description de singularités, mais de s'en saisir pour mieux les intégrer dans une actualité concrète. Or, c'est bien ce qu'effectuent, à l'appui de différents corpus, la plupart des auteurs.

Il est apparu que d'autres contributeurs, enfin, abordent l'interpellation dans une perspective principalement variationniste, même si leur démarche ne s'y arrête évidemment pas. Cette question de la variation n'indique pas que les recherches menées portent principalement sur des éléments de corpus discursifs (ce que font presque toutes les contributions au volume), mais qu'en plus de cela, elles insistent notamment sur les implications sociolinguistiques ou sociocognitives de l'interpellation, pour laquelle elles favorisent une démonstration empirique, à travers par exemple des "habitudes » linguistiques, voire ce qu'on pourrait appeler le « sens en pratique ».

Dans la droite ligne de ce qui a conduit certains acteurs de la recherche à réfléchir sur les enjeux de l'interjection, y compris sur le plan modal avec Liana Pop, laquelle parle d'un " mode pragmatique appellatif / vocatif ", de même que la répétition (journées d'études doctorales de Chambéry, avril 2007), la digression (Université d'El Manar, février 2006), la reformulation (Rennes 2, mai 2006), ou sur des problématiques plus restreintes telle que la périphérie gauche (Paris, CNRS, décembre 2006), les trente articles rassemblés ici constituent la première livraison d'actes d'une série de plusieurs manifestations, qui porteront sur les opérations discursives intempestives et sur l'instantané en discours, dans leur consistance à la fois épistémologique, ontologique et praxéologique. Dans cet esprit, et à travers la diversité qui les caractérise, les articles de cette édition ont, parmi leurs principaux mérites, celui de décrire les marqueurs, les expressions et les formes (et non pas strictement les formations) discursives auxquels renvoie l'interpellation, tout en la soustrayant de l'emploi qui en est effectué dans le contexte juridique (ou plus exactement judiciaire). 


\section{Présentation des contributions}

9 Il a été passionnant, au fur et à mesure des évaluations, et à un moment où le Comité Scientifique a joué son rôle non pas de tri ou de refus, mais de consolidation et de cadrage, de voir se dresser les contours à la fois ontologique et typologique (pour certains prévus, pour d'autres beaucoup moins), d'une interpellation qui s'ouvre à tous les possibles dans le cercle d'un dialogue ouvert. Ce dialogue s'est établi entre les champs disciplinaires bien sûr, mais aussi les approches méthodologiques, les objectifs de recherche, à la fois dans ce qui les distingue et dans ce qui constitue leurs analogies et leurs complémentarités.

10 Ainsi sont sollicités dans ce volume les liens entre interpellation et déixis (Auger, Fracchiolla, Moïse et Schultz-Romain / Détrie / Devriendt / L.S. Florea / Pop / Cini), ainsi que ceux qui s'établissent entre des éléments discursifs variablement mémorisés, et plus ou moins construits (en parataxe et en apotaxe par exemple). Les contributeurs nous invitent dans le même temps à mesurer l'enjeu de caractéristiques d'ordres prosodique (Bodelot et Verdier / Leon Miranda), acoustique (Fauré / Brouté), kinésique (Brouté / Leon Miranda), et, de manière tout à fait complémentaire, reviennent sur les questions de l'intensité (Auriac et Blasco-Dulbecco / Cabasino / M.L. Florea), de l'extraposition (Barbu / Vedrenne), du détachement (Doquet-Lacoste / Cini / Lehmann), avec cette possibilité de traiter les interpellatifs comme des éléments extraprédicatifs (Devriendt) ou dans le champ de l'adlocution (Bouchard).

11 Qu'il s'agisse d'en démontrer la dimension principalement allocutive (Détrie / Barbu), polylogale (Bouchard), ou encore inter et intralocutive (Biagioli / Fauré), notamment quand elle est envisagée en lien avec les vocalisations spontanées, l'interpellation se distingue des seuls appellatifs, et prend par exemple la forme d'interjections (Cini / Fauré) ou de questions toniques (Lailler). Saisie dans ce qui la rapproche de la nomination et de la dénomination (Détrie / Cabasino), elle paraît d'autant plus commune qu'elle s'immisce aussi facilement dans la célébration (Dumas), la commémoration (M.L. Florea), la convocation (Hammer), que dans la provocation (Auger, Fracchiolla, Moïse et SchultzRomain / Guilbert) et dans la mise en demeure (Mellet).

12 Les auteurs reviennent pour beaucoup sur les rapports entre les places occupées dans la hiérarchie sociale et familiale (Auger, Fracchiolla, Moïse et Schultz-Romain / Cabasino / Devriendt / Lehmann / Vedrenne), mais aussi sur la complexité de certaines connotations (Cabasino / Hammer). D'autre part, en plus du fait qu'elle peut être abordée à travers des supports filmiques (Devriendt / Cini) ou radiophoniques (Leon Miranda), on remarque avec les auteurs que l'interpellation occupe les textes liturgiques (Dumas), nécrologiques (M.L. Florea), romanesques (L.S. Florea), de même que le débat (Mellet), le commentaire (Guilbert), le faire-part (Hammer), la profession de foi (Rigat), les comédie et tragédie (Roesch), ainsi que l'épigramme (Vedrenne).

13 À cet ensemble d'approches et de corpus fait écho une certaine pluralité de perspectives, dont témoigne la sollicitation d'une "grammaire » interactive (Lailler / Fauré), de la rhétorique (Roesch), ainsi que le recours à une démarche comparatiste ou historicocomparative (Barbu / Cini / Lehmann / Orlandini et Poccetti / Roesch / Vedrenne), lesquelles s'avèrent assez bien représentées. Outre les similitudes qui interviennent entre certaines contributions, c'est bien la multidimensionnalité de l'objet interpellation que 
cette manifestation scientifique, qui s'est tenue à la Maison de la Recherche de la Sorbonne (Paris), les 16 et 17 mai 2008, a donc permis d'établir et / ou de conforter.

Envisagée à travers ce qui la contextualise (Doquet-Lacoste / Paveau / Biagioli / Bouchard), la conditionne (Brouté), l'annule éventuellement (Pop), l'interpellation s'inscrit selon les contributions dans une problématique de représentation (Paveau / L.S. Florea), d'allogénèse (Fauré), de professionnalité (Bouchard / Biagioli / Bernié), d'identité (Martinez / Rigat), ou encore incite les auteurs à l'intégrer dans une philosophie du discours (Paveau), dans un dialogue interculturel (Bouchard / Barbu), voire dans une épistémologie de l'existence (Guilbert).

En termes d'approches plus spécifiques enfin, on notera que les contributeurs traitent d'univers de référence fictionnels (L.S. Florea), d'idéologie (Paveau), tout en rappelant que l'interpellation se caractérise à la fois dans la manière qu'elle a de désigner l'interlocuteur bien sûr (Auger, Fracchiolla, Moïse et Schultz-Romain / Détrie / DoquetLacoste / Brouté / L.S. Florea), mais aussi de compenser le déficit d'interlocuteurs (Orlandini et Poccetti). Simultanément, d'autres schémas apparaissent, lesquels relèvent peut-être d'autres démarches explicatives, et correspondent ici à des phénomènes de ritualisation (Paveau), de co-construction (Devriendt), de parcours figuratif (Cabasino) ou de relais interpellatif (Pop).

Une telle diversité a conduit les organisateurs du Colloque à répartir les sessions suivant les constructions qui semblaient se dessiner. En revanche, il a été convenu, d'une part, de ne pas les thématiser (ce qui convient peu à l'esprit de pluridisciplinarité qui détermine en partie l'identité de ce type de manifestations), et, d'autre part, de ne pas les intégrer dans des cadres pré-établis. Les sessions, à vrai dire, se sont donc organisées en partie d'elles-mêmes : une première journée avec deux sessions qui ont dressé les premiers éléments d'une typologisation, et deux autres sessions qui, plaçant l'interpellation dans un cadre plus empirique, en ont dégagé les enjeux linguistiques et didactiques d'un côté, journalistiques et politiques de l'autre.

17 Une deuxième journée, qui a été l'occasion de réfléchir sur les stratégies discursives dont témoigne l'interpellation (ou la non-interpellation), a permis aux participants de revenir sur la dimension épistémologique de ces questions de premier plan. Les sessions ont également permis de questionner les opérateurs linguistiques de l'interpellation, tout en resituant cette dernière par rapport aux questions de l'allocution, de la nomination, des interjections, de l'apostrophe et de la convocation, avec, en fin de parcours, une présentation de ses enjeux sociologiques et historiques.

18 Les différentes sections du présent volume tentent, avec ce que les regroupements contiennent forcément de maladresses, de rassembler d'abord les articles qui permettent à la fois de cerner les principales caractéristiques de l'interpellation, et de montrer dans quelle mesure se complètent les démarches analytiques mises en œuvre. Elles regroupent ensuite les productions qui s'attachent tout particulièrement à la représentativité de certaines formules, ainsi qu'à la matérialité même des éléments et des contextes pris en compte. Les deux autres sections nous conduisent surtout, parmi d'autres possibilités, à en envisager la diversification : d'une part d'après les formes discursives et de textualité dans lesquelles apparaît l'interpellation, et d'autre part d'après les variations qui l'accompagnent.

19 L'un des points communs, parmi d'autres, qui ressortent des contributions, est celui qui consiste à démontrer le caractère instantané, mais aussi en partie irréversible de 
l'interpellation, et notamment à pointer le fait que, comme l'écrit Levinas (1991, 257), "de toute éternité un homme répond à un autre », mais dans un "appel » de ce qui s'opère « d'unique à unique ». Or, il nous semble opportun de rappeler qu'il s'en explique ainsi :

Appel du visage du prochain qui, dans son urgence éthique, ajourne ou efface les obligations que le "moi interpellé » se doit à lui-même et où le souci de la mort d'autrui peut pourtant importer au moi avant son souci de moi pour soi. L'authenticité du moi, ce serait cette écoute de premier appelé, cette attention à l'autre sans subrogation et, ainsi, déjà, la fidélité aux valeurs en dépit de sa propre mortalité.

Mais il n'est pas forcément dans notre propos de rapporter un point de vue particulier sur l'interpellation. Une présentation d'ouvrage n'étant pas une tribune, qu'on nous permette de laisser toute autre forme d'explication à la discrétion des auteurs.

\section{BIBLIOGRAPHIE}

Achard, P. (1995). Formation discursive, dialogisme et sociologie. Langages 117, 82-95.

Benveniste, E. (1977 [rééd.]). Problèmes de Linguistique générale. Paris : Gallimard.

Charaudeau, P. (2002). Grammaire du sens et de l'expression. Paris : Hachette.

Courtine, J.J. dir. (1981). Analyse du discours politique. Paris : Langages 62.

Détrie, C. (2007). De la non-personne à la personne : l'apostrophe nominale. Paris : CNRS éditions.

Forsgreen, M., Jonasson, K., Kronning, H. éds. (1998). Prédication, assertion, information, Actes du Colloque d'Uppsala en linguistique française (6-9 juin 1996). Uppsala : Acta Universitatis Upsaliensis.

Hammermüller, G. (1997). « Fräulein » oder « Frau » - « Menina » depois de casar ? Linguistica contrastiva. Deutsch versus Portugiesisch-Spanisch-Französisch, Hg.

Haroche, P., Henry, P., Pêcheux, M. (1971). La Sémantique et la coupure saussurienne : langue, langage, discours. Langages 24, 93-106.

Jacques, F. (2007). L'Arbre du texte et ses possibles. Paris : Vrin.

Lagorgette, D. (2003). Termes d'adresse, insultes et notion de détachement en diachronie. Cahiers de praxématique, 40, 43-69.

Lambrecht, K. (1998). Sur la Relation formelle et fonctionnelle entre topiques et vocatifs. Langues, 1-1, John Libbey Eurotext.

Levinas, E. (1991). Entre nous. Paris : Grasset.

Lopez-Muñoz, J. M., Marnette, S., Rosier, L. éds. (2004). Le Discours rapporté dans tous ses états : question de frontières? Paris : L'Harmattan.

Mayaffre, D. (2004). Formation(s) discursive(s) et discours politique : l'exemplarité des discours communistes versus bourgeois. Revue Texto ! [En ligne], URL : http://www.revue-texto.net/ index.php?id=585. 
Morrison, C.A., Ellis, A.W. (1995). Roles of word frequency and age of acquisition in word naming and lexical decision. Journal of Experimental Psychology : Learning, Memory and Cognition, 21, 116-133. Neveu, F. (2000). Des Noms - Nomination, désignation, interprétations. Paris : SEDES.

Noailly, M. dir. (1995). Noms propres et nomination. Paris : Klincksieck.

O'Kelly, D. dir. (2005). Nomination, Noms propres, termes d'adresse. Tomes XXVI-1/2, volumes 51 et 52 de Modèles Linguistiques, Toulon, USTV.

Pop, L. (2000). Espaces discursifs : pour une représentation des hétérogénéités discursives. Paris : Peeters.

Rastier, F. (1998). Prédication, Actance et Zones anthropiques. In M. Forsgren, K. Jonasson et H. Kronning (éds), Prédication, Assertion, Information, Acta Universitatis Uppsaliensis (Studia Romanica Uppsaliensa), Stockholm, Almquist et Wiksell International 56, 443-461.

Sassier, M. (2008). Genre, registre, formation discursive et corpus. Langage et Société 124/2, 39-57. Schegloff, E.A. (1979). Identification and Recognition in telephone openings. In G. Psathas (éd.), Everyday Language, New York : Erlbaum, 23-78.

Spieler, D.H., Balota, D.A. (2000). Factors influencing word naming in young and older adults. Psychology and Aging, 15, 225-231.

Stivers, T., Enfield, N.J. éds. (2007). Person Reference in Interaction : Linguistic, Cultural, and Social Perspectives. Cambridge : Cambridge University Press.

Vion, R. (1999). Pour une approche relationnelle des interactions verbales et des discours. Langage et Société 87, 95-114.

\section{AUTEURS}

\section{FRÉDÉRIC TORTERAT}

Université de Nice / IUFMEA 4080 (Paris-Sorbonne) / URE 03 (Nice)

\section{ANDRÉ THIBAULT}

Université de Paris-SorbonneEA 4080 (Paris-Sorbonne) 